\title{
Factors that impact implementation of mentorship programs in nursing academia: $A$ sequential-explanatory mixed methods study
}

\author{
Lorelli Nowell , Deborah White, Karen Benzies, Patricia Rosenau \\ University of Calgary, Calgary, Alberta, Canada
}

Received: March 10, 2017

Accepted: April 4, 2017

Online Published: April 13, 2017

DOI: $10.5430 /$ jnep.v7n10p1

URL: https://doi.org/10.5430/jnep.v7n10p1

\begin{abstract}
Objective: Nursing education institutions globally have called for mentorship to help address the nursing faculty shortage; however, little is known about the current state of mentorship or the barriers and facilitators for implementing mentorship programs in Canadian nursing schools. The objective of this study is to identify the current state of mentorship in Canadian nursing academia and explore factors that impact implementation of mentorship programs.

Methods: A sequential-explanatory mixed methods design with 2 phases: (1) cross sectional survey of nursing faculty to identify the current state of mentorship in Canadian schools of nursing and explore facilitators and barriers to implementing mentorship programs; and, (2) qualitative interviews about nursing faculty member's experiences with mentorship. Integration occurred when selecting phase 2 participants based on phase 1 results. A web-based survey was developed based on a review of literature; surveys were collected between September 2015 and March 2016 from 935 nursing faculty members. Survey participants $(n=48)$ were purposively sampled for maximum variation to participate in qualitative interviews, and data were analyzed thematically. Results: The majority of Canadian nursing schools lack formal mentorship programs with existing mentorship programs varying in scope and components. Factors influencing mentorship program implementation include: mentorship training and guidelines; quality of the mentoring relationships; choice and availability of mentors; organizational support; time and competing priorities; culture of the institution; and evaluation of mentorship outcomes.

Conclusions: Our results confirm lack of formalized mentorship programs in Canadian schools of nursing. In developing mentorship programs, academic leaders need to consider multiple barriers and facilitators to ensure success.
\end{abstract}

Key Words: Mentorship, Nurses, Nursing, Academia, Mixed methods study, Higher education

\section{INTRODUCTION}

Globally, nursing education programs are facing challenges in relation to the shortage of registered nursing faculty. ${ }^{[1]}$ In 2013, the World Health Organization described the shortage of nurse faculty in the majority of its member states as a key concern in the provision of human resources for healthcare. ${ }^{[2]}$ As the number of nurses in the workforce continues to decrease, so does the need for nursing faculty required to teach new nurses, study health problems, address patient issues, and inform health policy. This shortage of qualified nursing faculty is an issue across jurisdictions, and one that is anticipated to worsen if solutions are not found. ${ }^{[3]}$

Mentorship has been identified as one way to address the nursing faculty shortage by encouraging registered nurses

\footnotetext{
*Correspondence: Lorelli Nowell; Email: lnowell@ucalgary.ca; Address: University of Calgary, 2500 University Drive NWCalgary, AB, T2N 1N4, Canada.
} 
to enter and remain in nursing faculty roles. ${ }^{[4-7]}$ Confusion exists regarding the nature, definitions, and role of mentorship within nursing and across the broader literature. For the purpose of this study, mentorship is defined as:

A process whereby an experienced, highly regarded, empathetic person (the mentor) guides another (usually younger or more junior) individual (the mentee) in the development and reexamination of their own ideas, learning, and personal and professional development. The mentor, who often (but not necessarily) works in the same organization or field as the mentee, achieves this by listening or talking in confidence to the mentee. ${ }^{[8]}$

In a recent systematic review, mentorship had positive effects on behavioural, attitudinal, relational, motivational, and career outcomes of nurses working in academic settings. ${ }^{[9]}$ Despite the call for mentorship and the suggestion of positive outcomes associated with mentorship in nursing academia, the current state of mentorship in schools of nursing and the factors that impact the implementation of mentorship remains unclear. This scarcity of evidence is regrettable because it is needed for nursing schools to implement effective mentorship programs.

\subsection{Background}

In order for schools of nursing to be able to implement ef fective mentoring programs the current state of evidence for mentorship in nursing schools must be assessed. The barriers and facilitators to implement mentorship programs must be identified to support the design, implementation, and evaluation of mentorship programs. To the best of our knowledge, no studies have been conducted to understand current mentorship practices in nursing academia and factors that influence mentorship success.

A pragmatic worldview was used to underpin this mixed methods research study. A pragmatic viewpoint offers epistemological justification for bringing together multiple sources of knowledge with the goal of finding workable solutions, gaining a greater understanding of world in which we practice, and solving social problems. ${ }^{[10]}$ Pragmatism is not committed to one system of philosophy; therefore, drawing on this worldview allowed us to draw liberally from quantitative and qualitative assumptions, methods, techniques, and procedures to best meet the research needs and purposes. ${ }^{[11,12,12-14]}$

\subsection{Aims}

The overarching aims of the study were to: 2
(1) Identify the current state of mentorship in Canadian nursing academia, and

(2) Explore the facilitators and barriers to implementing mentorship programs.

\section{Methods}

\subsection{Design}

A sequential-explanatory mixed methods design ${ }^{[12]}$ including: (1) cross sectional survey of nursing faculty to identify the current state of mentorship in nursing academia and identify the facilitators and barriers to implementing mentorship programs, and (2) qualitative interviews with selected nursing faculty exploring their experiences with mentorship. Integration occurred when selecting phase 2 participants based on phase 1 results. The interview protocol was developed based on the results from phase 1 with the aim of investigating survey results in more depth. The results of both phases were integrated to develop a more robust and meaningful picture of mentorship.

\subsection{Setting/Context}

Within the Canadian Association of Schools of Nursing (CASN) are 81 English-speaking schools of nursing and 2,284 permanent faculty members spread over four regions. Of these schools, $28.9 \%$ offered one or more master's programs and $14.0 \%$ offered doctoral programs, in 20132014. ${ }^{[15]}$

\subsection{Ethical considerations}

Permission to conduct this research was granted through the local university Conjoint Health Research Ethics Board (REB15-0194). Arrangements were made to meet research governance requirements at individual schools of nursing.

\subsection{Phase 1: Online survey}

Cabana et al. ${ }^{[16]}$ classified generic barriers to program implementation into common themes that affected knowledge, attitudes, and behavior. This framework was further expanded in a systematic review conducted by Légaré et al. ${ }^{[1]}$ to include both barriers and facilitators. Applying this evidence based conceptual framework to this study helps illuminate the facilitators and barriers to implementing mentorship programs in schools of nursing and identify important variables and their potential relationships.

\subsubsection{Sample/Participants}

Participants were recruited from 81 schools of nursing through the CASN newsletter list serve and publically accessible email addresses. Inclusion was limited to faculty members of English speaking Canadian schools of nursing. A link to the online survey was included in the monthly

ISSN 1925-4040 E-ISSN 1925-4059 
CASN newsletters (September and October 2015) and sent to approximately 1,500 nursing academics across Canada. We estimated a $30 \%$ response rate $(n=685)$ to our online survey. ${ }^{[18]}$ The response rate $(n=98)$ from the first strategy was insufficient so we sent a detailed cover letter, consent form, and survey link to potential participants via publically accessible email addresses. The second wave of recruitment yielded a total of 837 additional responses for a final response rate of $40.9 \%(n=935)$.

\subsubsection{Data collection}

Similar to methods described by Rattray and Jones, ${ }^{[19]}$ the survey was designed by the researchers based on an integrative review of the literature. The review findings were mapped to the conceptual framework created by Légaré et al. ${ }^{[17]}$ to inform the development of the survey. To determine the face validity of the survey, preliminary review was sought from six nursing faculty members with mentorship experience from various schools of nursing in Canada. Reviewers were asked if the directions and statements were clear, adequately reflected the literature, and if any items needed to be revised or deleted. The revised survey was sent back to these reviewers to ensure concerns were adequately addressed. The survey consisted of 13 items including multiple choice and open-ended questions soliciting details of current mentorship programs, barriers and facilitators to the development of mentorship programs, and demographic information. Participants were also invited to provide written comments about additional mentorship components, mentorship evaluation, as well as barriers and facilitators. At the end of the survey, participants were asked to provide their email addresses if they were willing to participate in a follow up interview.

All data were collected between September 2015 and March 2016 using an electronic survey, supported by Fluid Survey software. ${ }^{[20]}$ The survey took approximately 5-10 minutes to complete. A detailed cover letter explaining why participant responses were important was included in the survey and reminder emails, during the second phase of recruitment, were sent at 2 and 4 weeks. ${ }^{[18]}$

\subsubsection{Data analysis}

Survey data were exported from Fluid Survey ${ }^{[20]}$ into excel, cleaned, and then imported into SPSS (version 22) statistical software package. Descriptive statistics included frequencies and percentages. Written responses to open ended questions were coded and thematically analyzed. ${ }^{[21]}$

\subsection{Phase 2: Qualitative interviews \\ 2.5.1 Sample/Participants}

Participants who agreed to be interviewed were purposively sampled based on survey results and selected to capture

Published by Sciedu Press variation across rank and tenure including professoriate and instructors, $\mathrm{PhD}$ and masters prepared faculty, and those who focus on graduate and undergraduate education $(n=48)$. Variation in school sizes, areas within Canada, and those with and without mentorship programs were sought.

\subsubsection{Data collection}

Development of the semi-structured interview guide was based on the results from the survey. The interview guide consisted of open-ended questions designed to explore the participant's perspectives and involvement with mentorship and barriers and facilitators to mentorship implementation. Individual, semi-structured telephone and face-to face interviews were conducted by a female doctoral candidate (LN) and were between 30 and 45 minutes long. Interviews were digitally recorded and transcribed verbatim. Field notes following the interviews captured researcher insights about mentorship. Data collection continued until saturation was achieved. $^{[22]}$

\subsubsection{Data analysis}

The process of qualitative data collection and analysis occurred iteratively. ${ }^{[12]}$ Transcripts and researcher notes were assigned unique identifiers and imported into NVivo (version 10) to support the organization and coding of the data. A six step thematic analysis, as outlined by Braun and Clarke, ${ }^{[23]}$ guided an iterative and reflective process involving a constant moving back and forward between each of the six steps. Themes elicited from participant qualitative interviews were categorized into barriers and facilitators to mentorship.

\subsubsection{Rigour}

Data collection, integration, and prolonged engagement with the data were used to increase the probability of credible findings being produced. ${ }^{[24]}$ To achieve dependability we ensured our research process was logical, traceable, and clearly documented by creating an audit trail. ${ }^{[25,26]}$ All records of raw data, field notes, transcripts, and a reflexive journal to were kept to help systemize, relate, and cross reference data, as well as ease the reporting of the research process. ${ }^{[27]}$ The researchers (LN, DW, KB, PR) met frequently to establish authenticity and trustworthiness of the research, and to insure interpretations were clear to the readers and true to the data.

\section{RESULTS}

\subsection{Characteristics of participants}

In phase 1, there were 935 respondents and 851 completed surveys for a response rate of $40.9 \%$, and a completion rate of $91 \%$. In phase 2, 48 participants participated in semistructured interviews. See Table 1 for characteristics of participants. 
Table 1. Characteristics of participants in Phase 1 and Phase 2

\begin{tabular}{|c|c|c|c|c|}
\hline \multirow{2}{*}{ Characteristics } & \multicolumn{2}{|c|}{ Phase $1\left(n=851^{*}\right)$} & \multicolumn{2}{|c|}{ Phase $2(n=48)$} \\
\hline & $n$ & $\%$ & $n$ & $\%$ \\
\hline \multicolumn{5}{|l|}{ CASN region } \\
\hline WNRCASN & 533 & 63 & 29 & 60 \\
\hline COUPN & 231 & 27 & 12 & 25 \\
\hline QRCASN & 18 & 2 & 1 & 2 \\
\hline ARCASN & 66 & 8 & 6 & 13 \\
\hline \multicolumn{5}{|l|}{ Number of faculty in the school } \\
\hline Less than 50 & 321 & 38 & 24 & 50 \\
\hline More than 50 & 311 & 37 & 19 & 40 \\
\hline I don't know & 214 & 25 & 5 & 10 \\
\hline \multicolumn{5}{|l|}{ Nursing programs offered } \\
\hline Bachelor’s degree only & 346 & 41 & 26 & 54 \\
\hline Graduate degrees in nursing & 505 & 59 & 22 & 46 \\
\hline \multicolumn{5}{|l|}{ Academic rank } \\
\hline Instructor Rank & 445 & 56 & 16 & 33 \\
\hline Professor Rank & 297 & 37 & 26 & 54 \\
\hline Leadership & 57 & 7 & 6 & 12 \\
\hline \multicolumn{5}{|l|}{ Highest level of education } \\
\hline Bachelor's degree & 148 & 17 & 2 & 4 \\
\hline Master's degree & 429 & 51 & 22 & 46 \\
\hline $\mathrm{PhD}$ & 236 & 28 & 24 & 50 \\
\hline Graduate degree in progress & 34 & 4 & 0 & 0 \\
\hline \multicolumn{5}{|l|}{ Years of experience } \\
\hline 0-4 years & 320 & 38 & 19 & 39 \\
\hline 5-9 years & 251 & 30 & 10 & 21 \\
\hline $10-14$ years & 150 & 18 & 9 & 19 \\
\hline More than 15 years & 128 & 16 & 10 & 20 \\
\hline \multicolumn{5}{|c|}{ Mentorship program in the school } \\
\hline Yes & 299 & 35 & 18 & 37.5 \\
\hline No & 550 & 65 & 30 & 62.5 \\
\hline
\end{tabular}

Note. ${ }^{*}$ missing values for some items therefore totals may not add to 851; WNRCASN = Western and Northern Region Canadian Association of Schools of Nursing; COUPN = Council of Ontario University Programs in Nursing; QRCASN = Quebec Region Canadian Association of Schools of Nursing; ARCASN = Atlantic Region Canadian Association of Schools of Nursing

\subsection{Phase 1: Results}

Of the 851 participants, only $35 \%$ had formal mentorship programs. When comparing results from across Canada, the WNRCASN had the highest percentage of respondents indicating they had a formal mentorship program $(41 \%)$, followed by the QRCASN (33\%), COUPN (28\%), with the lowest percentage occurring in ARCASN (21\%). Chi-squared tests revealed no statistically significant associations between the size of school and presence of mentorship program. A Chi-square test for independence (with Yates Continuity Correction) indicated significant association between schools of nursing with and without graduate programs, $\chi^{2}(1, n=849)$ $=8.559, p=.003$, phi $=0.103$. That is, compared to schools with graduate programs, schools with only undergraduate programs were more likely to have a formal mentorship program.

\subsubsection{Quantitative survey results}

Of the 299 participants (35\%) who indicated they had a mentorship program, respondents were asked to identify the components of their program using check boxes of components identified in the literature. See Figure 1 for components. Additional components identified in the open-ended questions included: new faculty being assigned a mentor; faculty development workshops; support for faculty to find their own mentor; and, mentorship coordinators.

Respondents were asked to rate the influence of facilitators and barriers to mentorship (see Figures 2 and 3). A number of participants (11\%-40\% dependent on the question) indicated they were unable to assess the facilitators and barriers to mentorship and of these participants, up to $79.3 \%$ responded they did not have a mentorship program. 


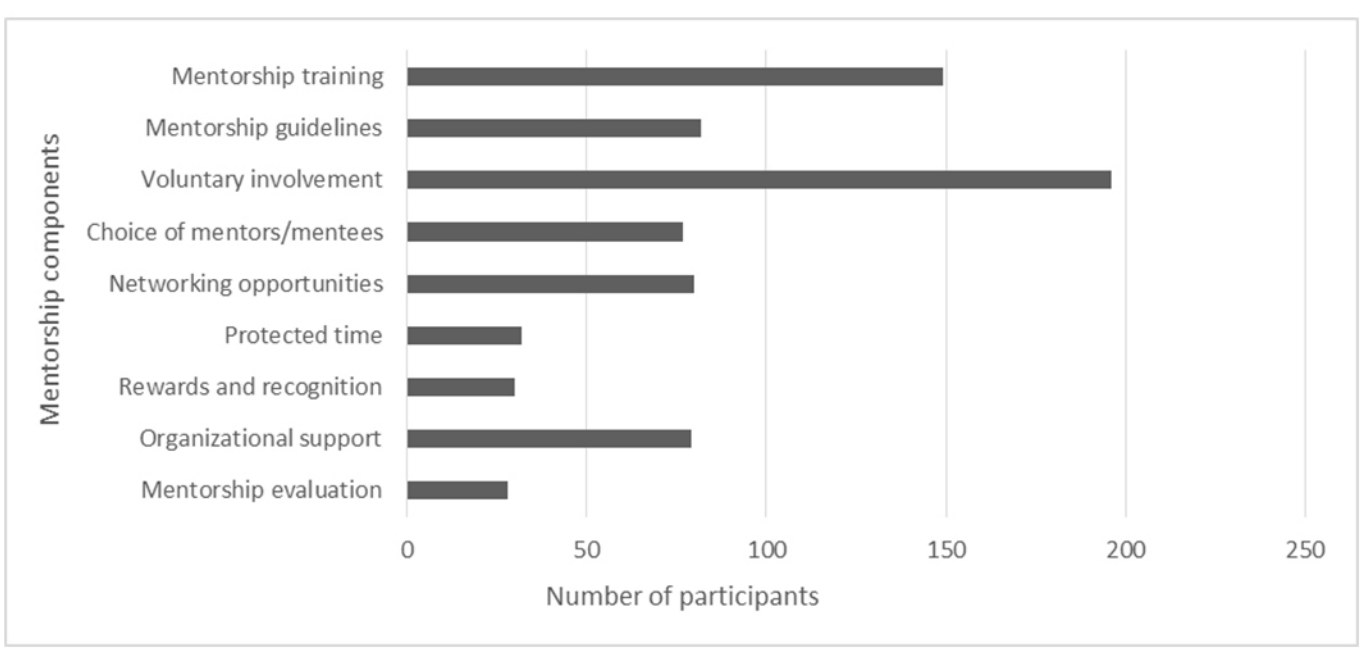

Figure 1. Mentorship program components

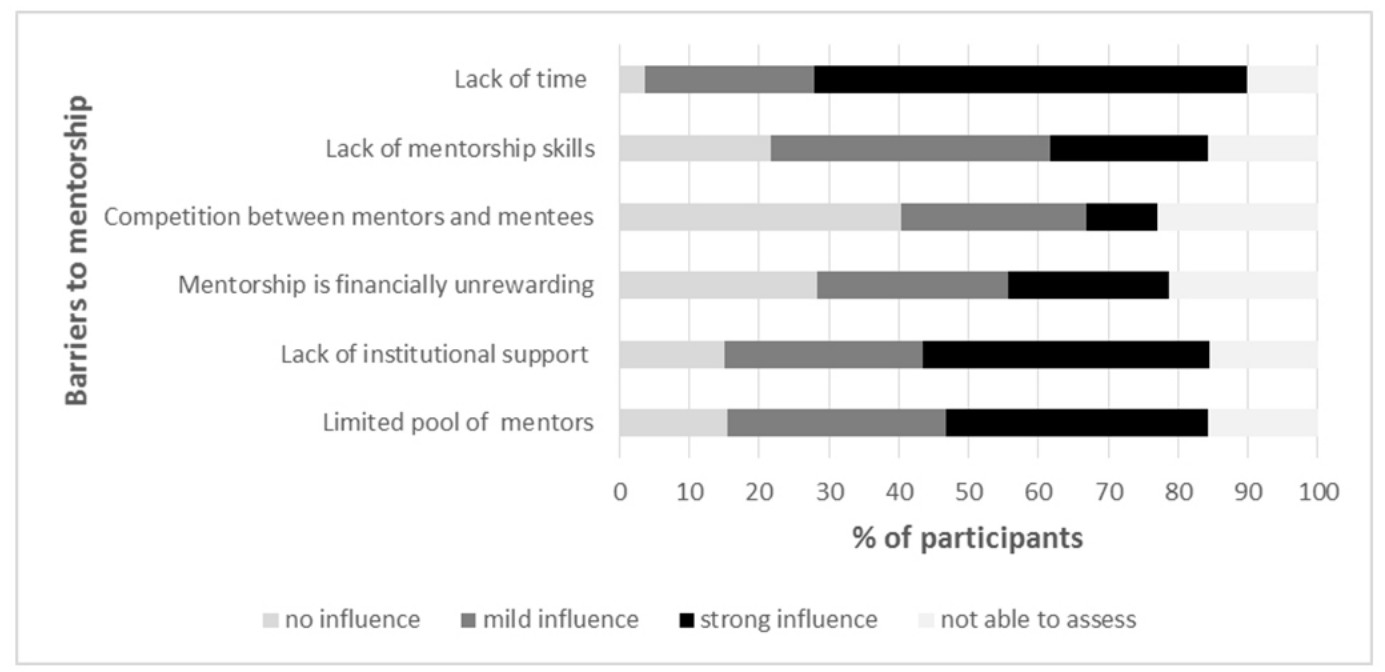

Figure 2. Participant responses to the influence of barriers to mentorship

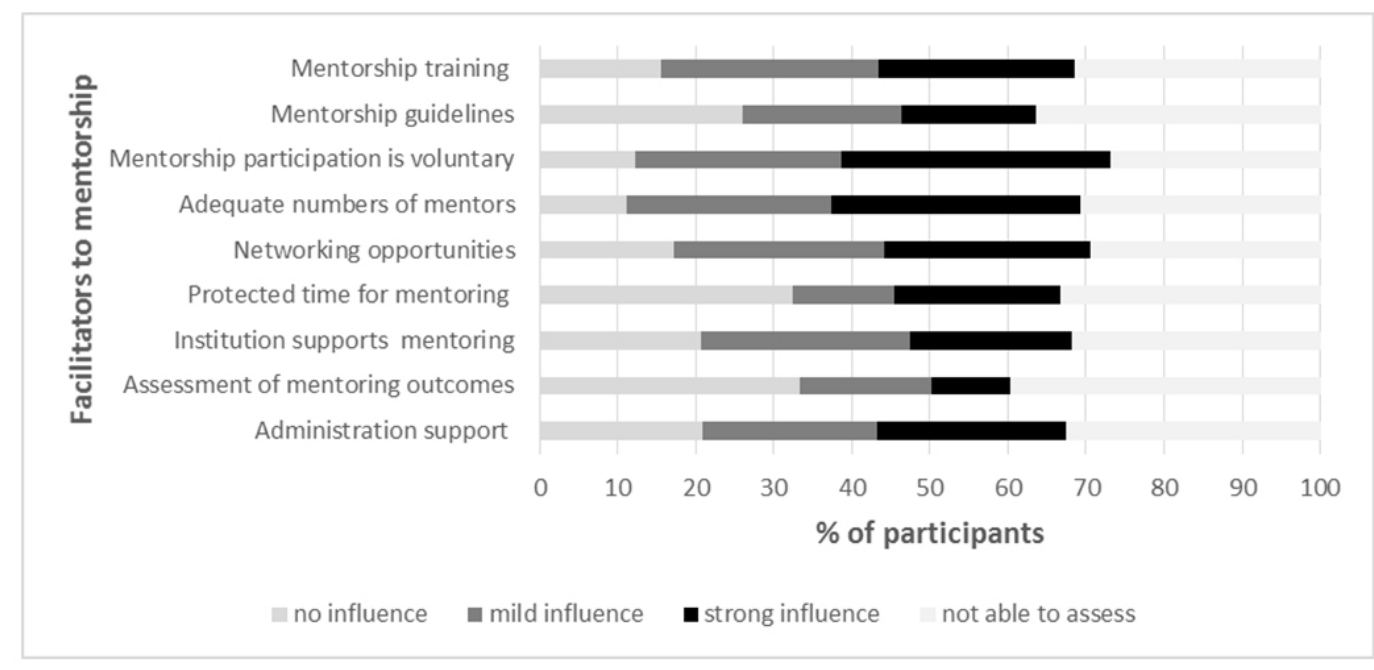

Figure 3. Participant responses to the influence of facilitators to mentorship 


\subsubsection{Open-ended questions survey results}

Additional facilitators to mentorship identified in the openended questions included: having a clearly identified need for mentorship; colleagues being open to mentorship; opportunities for inter-professional, interdisciplinary mentorship and distance mentorship; having a mentorship coordinator; and alignment with the nursing program vision and philosophy. Additional barriers to mentorship included: the lack of interest of potential mentors; high nursing faculty turnover; lack of resources; and, faculty culture.

\subsection{Phase 2: Results}

Within the barriers and facilitators themes, a number of subthemes emerged. These themes and subthemes are explored in further detail below.

\subsubsection{Barriers to mentorship}

\section{(1) Mentorship is not well defined}

Participants in this study issued a call for a common understanding of what mentorship means. "What does it look like? Who is involved? What's involved ... so that we're all... on the same page? Mentorship is often confused with other roles such as orientation and preceptorship and has become a bit of a buzz word. .. but that's not really what we're talking about" (P40). Others mentioned that the term mentorship was used to satisfy certification bodies but real mentorship was not actually taking place: "the intentions of my Dean is more to say on a CASN accreditation that we have a mentorship program for faculty, as opposed to really creating a culture of support and mentorship for our new faculty members" (P6).

\section{(2) Vulnerability of mentees}

Academic settings can be intimidating and new faculty members often feel vulnerable. Participants described feeling "there's an implied level of self-sufficiency, based on the fact that you kind of got where you got to. It's almost like well, you should be able to figure it out" (P37). New faculty members described wanting to demonstrate their competence and capacity, and by acknowledging that they wanted or needed mentorship required some vulnerability.

\section{(3) Limited pool of mentors}

The limited pool of mentors was identified as being a significant challenge to implementing and sustaining mentorship programs. Participants suggested that the lack of mentors led to inconsistencies in the nursing faculty role "things were falling through the cracks... there was just too many new faculty and not enough mentors" (P8). The workload of mentorship was also related to the lack of available mentors "it's left to a smaller body of tenured fulltime faculty, who again, have way more responsibilities and taking on that piece of mentorship... involves a lot of time I think and it becomes really difficult" (P23). There was also a noted lack of interest from senior faculty in mentoring others "the matriarchs in the program, they're not interested in helping anybody else. They're just banging off the publications and getting as much credit for it as they can" (P31). This is further challenged by frequent faculty turnover and retirements.

\section{(4) Time, workload, and competing priorities}

Mentorship requires time-a limited resource. Almost all participants identified time, workload and competing priorities as a key challenge to implementing mentorship because "people really have to work hard at to be able to take somebody on and be willing to kind of help them through ... the complex elements of a faculty role" (P23). Academic pressures are becoming greater, and this limits the time available to dedicate to mentorship "the rules of the game of being a nurse academic have changed ... there's so much pressure in terms of research and getting grants and... being really active outside of your teaching role ... it's even more intense now" (P21). Suggestions on how to overcome this barrier included "time release for both parties, the mentee and the mentor and ... some development, so that there is a coherent mentorship that's consistent" (P22). Some nursing schools provided "a little bump in credits associated with... mentorship relative to scholarship, relative to teaching learning, relative to all the aspects that are sort of a part of your job" (P23). Others suggested a dedicated position for faculty mentorship was needed because "having somebody do this off the side of their desk does not work... having it as an add-on to people's responsibilities does not work" (P12). When a dedicated person is in charge of mentorship they "need a certain percentage of their time to be dedicated to learning and understanding that role" (P30), and "there has to be workload allocation for mentoring in somebody's workload, so that it's not perceived as they're draining you" (P32).

\section{(5) Lack of support from leadership}

Mentorship programs without allocated funding or programs that had undergone cutbacks appeared to struggle indicating that mentorship funding is "one of the things that we haven't been able to support in the manner that we should" (P15). Mentorship was cited as "something that management just doesn't look at and doesn't appreciate" (P17), and this had a significant impact on the mentorship culture. "The leadership of our department are not mentors themselves and so there is no culture of mentorship... they don't understand the role of mentorship... if the leadership doesn't create that culture and doesn't make it a normal part of the job and encourage it, then people get lost in their own life" (P25). Establishing a culture of mentorship requires "visionary, capable leaders as 
Directors and Deans... we don't have capable leaders who value mentorship, value nursing, value each other, and that's what's needed" (P16).

\section{(6) Culture of competitiveness}

The nature of academia is competitive and this has a negative impact on the ability to establish a culture of mentorship. "We're scrambling to get the next grant and competing with others, it's turning people into very competitive academics who do not want to share and mentor" (P16), and it "sets up a competition instead of a collaboration... it divides us. It doesn't enable us to move forward" (P35). A number of participants suggested that senior and successful academics often do not want to mentor junior faculty. "There were very senior people here who could have been mentors, but they were resentful, jealous, and competitive. So it's not like they weren't present... I don't think they wanted to see me succeed" (P16). Others described "turf wars or territorial wars" where "new faculty coming onboard may have a certain skill set or knowledge or expertise in an area that may be seen as competing with some of the faculty that are already onboard" (P24). Rather than collaborating, participants expressed it was better to work independently because "its best if you build silos because then you collect all the greatness for yourself... it's about having more merit than someone else and having more grant money, more publications, bigger $\mathrm{CV}$ and just being heralded as some sort of great guru" (P31).

\subsubsection{Facilitators for mentorship}

\section{(1) Mentorship training}

Mentorship training is an important aspect for implementing mentorship programs. Participant descriptions of mentorship training included "models of mentorship" (P10), "what mentoring relationships look like and how to make it work" (P17), and the importance of workshops to "talk about mentorship and just really encourage faculty to seek out mentorship relationships with each other" (P21). Mentorship training specifically for mentors was viewed as important "because people are thrown in to be a mentor and they don't have a clue what they're doing really" (P19).

\section{(2) Mentorship guidelines}

To ensure consistency and stability, mentorship programs "need to have some established, thought out guidelines and some evidence to support that what you're going to put together is valuable for the mentor and mentee" (P18). Having consistent guidelines "are always useful, so that it's not just hit or miss" (P14), and should include "a really clear job description and roles and what the person is responsible for" (P1) and "outline objectives and expectations really clearly" (P30).

Published by Sciedu Press

\section{(3) Networking opportunities}

Networking opportunities consistently had a positive impact on mentorship because they "provide more exposure... so you inherently have more opportunity to build and to find mentorship" (P37). Networking opportunities were often informal such as "being invited to meetings and even coffee chats with colleagues" (P46) and "exploring people's ideas for research" (P26) while others were more formal "opportunities throughout the year ... to meet with other new faculty" (P18). Suggestions for networking opportunities should be aimed at "sharing each other's success... what they've done, what they're bringing through sharing your own research and work that you've accumulated over the years so it becomes known what sort of a resource you are within the department" (P35).

\section{(4) Institutional support}

Funding allocation for mentorship is an important facilitator for establishing mentorship programs. One participant currently actively involved in mentorship noted, "administrative support and funding ... has been crucial" (P12). Institutional mentorship programs offered to all faculty were viewed as valuable. The perceived positive outcomes of these programs included bringing "new faculty together from across the different schools" (P25), and providing a place where "faculty can go and work with them there around looking at teaching and learning and how that might work" (P39) or a place that "faculty could go if they had research ideas and maybe working with other disciplines and how could they get that going" (P39).

\section{(5) Ongoing assessment and reporting of mentorship out- comes}

In order for mentorship to be successful and for the faculty to embrace it there needs to be clear mentorship goals that are "really focused in what the needs of the organization are" (P4). Having specific mentorship goals as a "part of your strategic plan or your plan for your department or your faculty" (P10) facilitates evaluation. Once goals have been established it is important to "take [measure] some hard outcomes ... publications, funded grants, participation on teams, supervision of graduate students, good teaching evaluations, participation on university committees, etc.” (P28). Evaluation was cited as being important to be able to "improve upon that whole process" (P21) and participants agreed, "there should be a follow-up... an evaluation. Was this [mentorship] effective? What are we doing right and what are we not doing right?" (P11) These data could then "be brought to the mentor committee for them to make changes" (P19). 


\section{(6) Qualities of the mentoring relationship}

Participants who had experience as mentees spoke about qualities they sought out in a mentor. Mentors were often chosen "based on their characteristics and their knowledge and their experience" (P43). Credible mentors were believed to be "absolutely passionate about their work and extremely excited about teaching" [and were] "a fabulous role model as to what a stellar exemplar educator should be like" (P29) as well as "someone that's emotionally intelligent and just very well respected" (P13). Carefully thought out mentorship matches and/or mentee and mentor choice in matching is required to ensure that mentors and mentees work well together because mentorship is "not something that can be effective if you just sort of introduce two people and then [say] you're the mentor and you're the mentee, go have at it' (P15). Mentorship matches also need to be made based on shared philosophy because "it's really hard to mentor somebody that you don't have a shared sort of understanding of ... what you think goes into nursing education and what's important relative to nursing" (P23). When mentorship matches were assigned without input from the participants, the relationships appeared to struggle because "when you force mentorship... you can also connect people who don't really get along" (P25). A further matching challenge was identified when mentors or mentees did not want to be part of a mentoring relationship. "There are people who don't want to be mentors. They don't see themselves as being mentors. They don't have the time perhaps or the interest and so, assigning them to be mentors would be a disaster" (P10).

Mentors and mentees also need to demonstrate commitment to the mentoring relationship. One of the greatest gifts a mentor can give a mentee is their time: "I really appreciate... they were willing to give time to me to listen to my ideas, engage with me about my ideas relative to teaching and learning and relative to the establishment of research" (P23). Mentoring relationships were frequently described as a reciprocal process. Many suggested that with mentorship it is the "give and take of it that keeps both of you refreshed" (P9). Reciprocal mentoring relationships allowed participants to connect "in a meaningful way that allows one to learn from the other" (P36), and this helped to "really built capacity" (P20). When mentorship was viewed as reciprocal there was "... more of a sense of equality ... and not a sense of hierarchy" (P42); however, when mentorship was "not reciprocal it becomes a burden" (P9). In order for these reciprocal relationships to work "you have to be able to trust each other, because otherwise ... there is always the danger of people feeling judged or evaluated and that cuts out that relationship" (P25). Therefore, mentors and mentees "should be someone that you can confide in, express your excitement to, your passion, your vulnerability, like all of those things. It should be a relationship that is genuine" (P38).

A number of participants spoke about having multiple mentors and appreciated "having opportunities to work with different faculty with different sets of skills" (P24) and "multiple people, who might be able to support people or mentor people in different things" (P25). It was important to acknowledge that mentorship "doesn't have to always be within the organization... having some other outside experts, who understand that academia role as well would be helpful" (P30).

\section{DiscuSSION}

The overarching aim of the study was to identify the current state of mentorship in nursing academia and explore the facilitators and barriers to implementing mentorship programs. Our findings suggest that despite the call for mentorship, many schools of nursing do not have formal mentorship programs; and the mentorship programs that are in place vary in their scope and components. A number of important factors to consider when developing and implementing mentorship in nursing academia were identified including: mentorship training and guidelines; qualities of the mentoring relationships; choice and availability of mentors; organizational support; time and competing priorities; culture of the institution; and, evaluation of mentorship outcomes.

Mentorship training and guidelines were a component of current mentorship programs. Participants identified training and guidelines were a substantial facilitator to mentorship, and the lack of guidelines and training a barrier. Mentorship training and guidelines have been acknowledged by others to be a significant facilitator for mentorship. ${ }^{[28,29]}$ Mentorship guidelines may be used to list and explain the mentor and mentee responsibilities, goals and expectations to help minimize misunderstandings. ${ }^{[30-32]}$ Mentorship training could focus on mentorship qualities, university expectations of mentors, communication skills necessary for effective mentoring, mentoring resources, stages of the mentoring relationship, how to find good mentors, prepare for mentorship meetings, and break up with a mentor. ${ }^{[33,34]}$ To ensure consistency and stability, we recommend that mentorship programs develop clear guidelines that outline objectives, expectations, and role descriptions as well as mentorship training to support both mentors and mentees in their roles.

Voluntary involvement in mentorship was the most frequent component of mentorship programs and was identified as a key facilitator to mentorship. Similarly, the qualitative interviews found that mentors and mentees needed to be com- 
mitted to mentorship. Authors of studies on academic mentorship have suggested that mentorship participation should be voluntary. ${ }^{[31,32]}$ Appropriate fit between mentors and mentees is an important aspect for creating a successful mentoring relationship, yet there is no consensus in the nursing literature on how and why mentors and mentees should be matched. Relationship quality may affect mentorship outcomes. ${ }^{[9]}$ Therefore, we recommend voluntary involvement in mentorship programs, and that mentors and mentees be included in the matching process.

Availability of mentors was identified as a facilitator, and the limited pool of available mentors was identified as an important barrier to mentorship. The success of mentoring programs are often limited by the availability of faculty to serve as mentors and the failure of the institution to identify a significant pool of potential mentors. ${ }^{[30,35,36]}$ The lack of diversity in interests and skills amongst senior faculty members in academia has also been identified as problematic as mentees are often unable to find suitable mentors. ${ }^{[32]} \mathrm{Al}-$ though a lack of mentors has been identified as a barrier to mentorship, strategies to meet the needs of mentees in terms of choice, variety, and style of mentorship have been identified. Participants in our study spoke about having multiple mentors, and appreciated having mentors outside of the organization and even the nursing profession. Mentees may require more than one mentor to address the wide range of mentorship needs, ${ }^{[30]}$ and they may find it beneficial to have several mentors for academic, scientific, and social needs. ${ }^{[36]}$ Potential mentors may be identified at other institutions ${ }^{[36]}$ and administrators should consider distance mentorship opportunities. ${ }^{[37]}$ Networking opportunities may provide important opportunities to support the development of mentorship matches. ${ }^{[5,38]}$ Furthermore, these networking opportunities may be helpful in recruiting faculty to serve as mentors in future mentoring programs. In light of the nursing faculty shortage, ${ }^{[1,2,39]}$ we recommend exploring mentorship opportunities outside of nursing academia to help maximize mentors' resources, allow mentees to experience mentors with various skills sets, and provide greater opportunities for network expansion.

Within our study, less than one third of participants who indicated they had a formal mentorship programs identified they had institutional support, and most noted lack of institutional support as a barrier to mentorship. Within the literature, lack of leadership, direction, and encouragement from administration to support mentors and mentees was recognized as a key barrier to mentorship in academia. ${ }^{[40,41]}$ For a mentorship program to be effective, faculty need to know their institution is actively supporting and facilitating the development of mentoring relationships. ${ }^{[42]}$ Universities, as well

Published by Sciedu Press as accreditation and quality evaluation agencies should be committed to faculty growth and development and should therefore provide adequate support to develop mentorship capacities. ${ }^{[43,44]}$ Others have also identified administrative support and commitment as being a primary component of an effective mentoring program, suggesting that without authentic support from administration, mentoring programs are likely to struggle. ${ }^{[5,35]}$ We recommend that leaders and administrators of nursing schools visibly declare their support for mentorship programs and help develop a clear vision for mentorship to direct, align, and inspire motivation and action from the greatest numbers of faculty members.

Protected time for mentoring activities was one of the least common mentorship program components, yet lack of time for mentorship was identified as the greatest barrier to mentorship. Almost all participants identified time, workload and competing priorities as a key challenge to implementing mentorship. The time required from both the mentor and mentee to engage in meaningful mentorship activities was the most frequently noted barrier to mentorship within the mentorship literature. ${ }^{[6,36,40,45,46]}$ Just as a lack of time for mentorship was a barrier, protected time for mentorship was a facilitator. Mentors and mentees who had protected time for mentorship viewed the commitment from their institution as a sign of acceptance and support for mentoring activities. ${ }^{[30,35,40]}$ In some mentorship programs, faculty received acknowledgment and credit for time spent mentoring, as demonstrated by promotion and tenure, salary merit criteria, as well as workload calculations. ${ }^{[5]}$ When designing and implementing mentorship programs, we recommend that consideration be given to time expectations and how they may encourage or discourage mentors and mentees to engage in mentoring activities.

Schools of nursing with undergraduate programs only were more likely to have formalized mentorship programs than schools of nursing with both undergraduate and graduate programs. Schools of nursing solely offering undergraduate education have a primary focus on teaching, with mentorship targeted towards faculty teaching development. Programs with both undergraduate and graduate programs balance both the teaching and research development of their faculty. This potentially makes the development of mentorship programs more complex to meet the various mentorship needs of the faculty. Furthermore, schools of nursing with graduate programs may foster a more competitive environment, which influences the ability to establish a culture of mentorship. Academia has a history of fostering a culture of competitiveness between mentors and mentees and this is a barrier to mentorship. ${ }^{[4]}$ The academic systems of promotion and tenure often reward faculty exclusively for funded research 
and publications, often at the expense of teaching and mentoring. ${ }^{[47,48]}$ A culture of mentorship can be facilitated by formally acknowledging mentoring as central to the philosophy of the faculty or school by educating faculty about the importance of mentoring. ${ }^{[6,48,49]}$ Cultural supports may include selection of departmental heads who are committed to facilitating mentorship through mentoring programs and committees, ${ }^{[49]}$ and support the development of a noncompetitive environment. ${ }^{[40]}$ When planning and implementing mentorship programs we recommend strategies to mitigate the culture of competitiveness recognizing that the current state of research funding potentially increases this competitive environment.

Decision makers often require clear evidence to support the implementation and continuation of programs such as mentorship; however, few mentorship programs have evaluation components and mentorship outcomes were not identified as important facilitators to mentorship. Similar to our findings, lack of evaluation is a well-documented gap in academic mentorship literature. ${ }^{[35,50]}$ Authors have recommended ongoing evaluation, including formative and summative evaluation, with defined mentorship benchmarks. ${ }^{[32,40]}$ Without rigorous evaluation of mentorship programs, it is challenging to draw conclusions regarding the achievement of specified goals. We recommend that mentorship programs are thoroughly assessed and mentoring-relevant outcomes are reported to highlight if mentorship programs are a worthy investment.

\section{Strengths and limitations}

This study is strengthened by the inclusion of participants from a diverse range of nursing schools and roles across
Canada. The sequential explanatory mixed methods design was especially useful as nationwide quantitative data was used to speak to the larger population while providing the depth of a qualitative study. ${ }^{[12,14]}$ The inclusion of a variety of schools allowed us to explore the complexity and variation of mentorship to achieve the fullest understanding of this concept, however, comparison with schools of nursing in other countries would strengthen the research. This study is limited by including only English-speaking participants. This study was undertaken within one country and may not be generalizable to other locations, where schools of nursing may vary from the Canadian context. Furthermore, participants were self-selected; however, participants for phase two of the study were purposively selected for maximum variation.

\section{Conclusion}

Nursing schools require nursing faculty to assume a complex role and a global call for mentorship has been issued. However, mentorship is a multifaceted phenomenon and a number of important factors may impact mentorship success. This study helps move the mentorship research agenda forward by describing the current state of mentorship and the factors that impact the implementation of mentorship strategies in nursing academia. The findings may be used by faculties of nursing to inform the development of future mentorship strategies that mitigate barriers and leverage facilitators with the aim of developing more successful mentorship programs.

\section{CONFLICTS OF INTEREST DISCLOSURE}

The authors declare that there is no conflict of interest.

\section{REFERENCES}

[1] Benner P, Sutphen M, Leonard V, et al. Educating nurses: a call for radical transformation. San Francisco: Jossey-Bass; 2010.

[2] World Health Organization. Transforming and Scaling up Health Professional Education and Training: Policy Brief on Faculty Development. Geneva, Switzerland: World Health Organization; 2013.

[3] Reid TP, Hinderer KA, Jarosinski JM, et al. Expert clinician to clinical teacher: Developing a faculty academy and mentoring initiative. Nurse Education in Practice. 2013; 13(4): 288-293. http: //dx.doi.org/10.1016/j.nepr.2013.03.022

[4] National League for Nursing. Mentoring of nurse faculty. National League for Nursing; 2006.

[5] Nick JM, Delahoyde TM, Prato DD, et al. Best practices in academic mentoring: a model for excellence. Nursing Research \& Practice. 2012: 1-9. http://dx.doi.org/10.1155/2012/937906

[6] Sawatzky J, Enns CL. A mentoring needs assessment: validating mentorship in nursing education. Journal of Professional Nursing 2009; 25(3): 145-150. http://dx.doi.org/10.1016/j.profn urs.2009.01.003

[7] Wyte-Lake T, Tran K, Bowman C, et al. A systematic review of strategies to address the clinical nursing faculty shortage. Journal of
Nursing Education. 2013; 52(5): 245-252. http://dx .doi .org/1 $0.3928 / 01484834-20130213-02$

[8] Straus S, Sackett DL. Mentorship in academic medicine. Chichester, West Sussex, U.K.: John Wiley \& Sons; 2014.

[9] Nowell L, Norris JM, Mrklas K, et al. Mixed methods systematic review exploring mentorship outcomes in nursing academia. Journal of Advanced Nursing. 2016; 73(3): 527-544. http://dx.doi.org /doi:10.1111/jan.13152

[10] Johnson BR, Onwuegbuzie AJ. Mixed methods research: A research paradigm whose time has come. Educational Researcher. 2004; 33(7): 14-26. http://dx.doi.org/10.2307/3700093

[11] Cherryholmes C. Notes on pragmatism and scientific realism. Educational Researcher. 1992; 21(6): 13-17. https://doi.org/10.310 2/0013189X021006013

[12] Creswell J. Research design: Qualitative, quantitative, and mixed methods approaches, 4th edn. Thousand Oaks, CA: Sage; 2014.

[13] Morgan DL. Paradigms lost and pragmatism regained: Methodological implications of combining qualitative and quantitative methods. Journal of Mixed Methods Research. 2007; 1(1): 48-76. http: //dx.doi.org/10.1177/2345678906292462 
[14] Tashakkori A, Teddlie C. Sage handbook of mixed methods in social science and behavioral research, 2 edn. Thousand Oaks, CA: Sage; 2010. https://doi.org/10.4135/9781506335193

[15] Canadian Association of Schools of Nursing: Registered Nurses Education in Canada Statistics 2013-2014 Registered Nurse Workforce, Canadian Production: Potential New Supply; 2015.

[16] Cabana M, Rand C, Powe N, et al. Why don't physicians follow clinical practice guidelines? A framework for improvement. The Journal of the American Medical Association. 1999; 282(15): 1458-1465. http://dx.doi.org/10.1001/jama.282.15.1458

[17] Légaré F, Ratté S, Gravel K, et al. Barriers and facilitators to implementing shared decision-making in clinical practice: Update of a systematic review of health professionals' perceptions. Patient Education and Counseling. 2008; 73(3): 526-535. http://dx.doi.o $\mathrm{rg} / 10.1016 / \mathrm{j} \cdot \mathrm{pec} .2008 .07 .018$

[18] Dillman D, Smyth J, Christian L. Internet, mail, and mixed-mode surveys: The tailored design method. Hoboken, New Jersey: John Wiley and sons; 2009.

[19] Rattray J, Jones MC. Essential elements of questionnaire design and development. Journal of Clinical Nursing. 2007; 16(2): 234-243. http://dx.doi.org/10.1111/j.1365-2702.2006.01573.x

[20] Fluid Survey [Computer software]. San Mateo. California, USA.

[21] Pallant J. A step by step guide to data analysis using IBM SPSS: SPSS survival manual, 5 edn. Berkshire, England: Open University Press; 2013.

[22] Miles M, Huberman A, Saldana J. Qualitative data analysis: A methods sourcebook., 3 edn. Los Angeles, CA: Sage; 2014.

[23] Braun V, Clarke V. Using thematic analysis in psychology. Qualitative Research in Psychology. 2006; 3(2): 77-101. http://dx.doi . org/10.1191/1478088706qp063oa

[24] Lincoln Y, Guba EG. Naturalistic inquiry. Newbury Park, CA: Sage; 1985.

[25] Tobin GA, Begley CM. Methodological rigour within a qualitative framework. Journal of Advanced Nursing. 2004; 48(4): 388-396. http://dx.doi.org/10.1111/j.1365-2648.2004.03207.x

[26] Koch T. Establishing rigour in qualitative research: The decision trail. Journal of Advanced Nursing. 1994; 19(5): 976-986. http: //dx.doi.org/10.1111/j.1365-2648.1994.tb01177.x

[27] Halpren ES. Auditing naturalistic inquiries: The development and application of a model. Indiana University; 1983.

[28] Allen TD, Eby LT, Lentz E. Mentorship Behaviors and Mentorship Quality Associated With Formal Mentoring Programs: Closing the Gap Between Research and Practice. Journal of Applied Psychology. 2006; 91(3): 567-578. PMid:16737355 https://doi.org/10.1 037/0021-9010.91.3.567

[29] Kram KE. Mentoring at work: Developmental relationships in organizational life. Boston, MD: University Press of America; 1988.

[30] Keyser D, Lakoski J, Lara-Cinisomo S, et al. Advancing institutional efforts to support research mentorship: A conceptual framework and self-assessment tool. Academic Medicine. 2008; 83(8): 217-225. http://dx.doi.org/10.1097/ACM.0b13e318163700a

[31] Schulze S. Mentees' views of a structured mentoring programme at Unisa. South African Journal of Higher Education. 2010; 24(5): 782-799.

[32] Zellers DF, Howard VM, Barcic MA. Faculty mentoring programs: Reenvisioning rather than reinventing the wheel. Review of Educational Research. 2008; 78(3): 552-588. http://dx.doi.org/10. $2307 / 40071137$

[33] Straus S, Johnson MO, Marquez C, et al. Characteristics of successful and failed mentoring relationships: A qualitative study across two academic health centers. Academic Medicine. 2013; 88(1): 82-89. http://dx.doi.org/10.1097/ACM.0b013e31827647a0

Published by Sciedu Press
[34] Byrne G, Topping A, Kendall S, et al. Developing a national mentorship scheme to enhance the contribution of clinical academics to health care. Nurse Researcher. 2014; 22(2): 23-28. PMid:25423938 https://doi.org/10.7748/nr.22.2.23.e1288

[35] Kashiwagi DT, Varkey P, Cook DA. Mentoring programs for physicians in academic medicine: A systematic review. Academic Medicine. 2013; 88(7): 1029-1037. http://dx.doi.org/10.10 97/ACM. Ob013e318294f368

[36] Straus S, Chatur F, Taylor M. Issues in the mentor-mentee relationship in academic medicine: A qualitative study. Academic Medicine. 2009; 84(1): 135-139. http://dx.doi.org/10.1097/ACM.0b0 $13 \mathrm{e} 31819301 \mathrm{ab}$

[37] Robertson TMA. Making the connection: How mentors choose proteges in academic mentoring relationships (Doctoral dissertation). Ann Arbor: University of North Texas; 2010.

[38] White A, Brannan J, Wilson CB. A mentor-protege program for new faculty, part I: stories of proteges. Journal of Nursing Education. 2010; 49(11): 601-607. PMid:20672778 https ://doi .org/10.3 928/01484834-20100630-04

[39] Institute of Medicine of the National Academies. The future of nursing: leading change, advancing health. United States of America: The National Academies Press; 2011.

[40] Hubbard C, Halcomb K, Foley B, et al. Mentoring: A nurse educator survey. Teaching and Learning in Nursing. 2010; 5(4): 139-142. http://dx.doi.org/10.1016/j.teln.2010.02.006

[41] Ehrich LC, Hansford B, Tennent L. Formal mentoring programs in education and other professions: A review of the literature. Educational Administration Quarterly. 2004; 40(4): 518-540. http: //dx.doi.org/10.1177/0013161x04267118

[42] Bickel J, Rosenthal S. Difficult issues in mentoring: Recommendations on making the "undiscussable" discussable. Academic Medicine. 2011; 86(10): 1229-1234. http://dx.doi.org/10.1097/ACM. 0 b013e31822c0df7

[43] Angulo LM, Alegre De La Rosa OM. Online faculty development in the canary islands: A study of e-mentoring. Higher Education in Europe. 2006; 31(1): 65-81. http://dx. doi .org/10.1080/037 97720600861243

[44] Chesler NC, Chesler MA. Gender-informed mentoring strategies for women engineering scholars: On establishing a caring community. Journal of Engineering Education. 2002; 91(1): 49-55. https://doi.org/10.1002/j.2168-9830.2002.tb00672.x

[45] Ehrich L, Tennent L, Hansford B. A review of mentoring in education: some lessons for nursing. Contemporary Nurse: A Journal for the Australian Nursing Profession. 2002; 12(3): 253-264. http://dx.doi.org/10.5172/conu.12.3.253

[46] Wilson C, Brannan J, White A. A mentor-protégé program for new faculty, part II: Stories of mentors. Journal of Nursing Education. 2010; 49(12): 665-671. http://dx.doi.org/10.3928/0148483 4-20100730-08

[47] Turnbull BJ, Roberts K. Nurse-academics' mentorship: Rhetoric or reality? Collegian: Journal of the Royal College of Nursing, Australia. 2005; 12(2): 33-38. http://dx.doi.org/10.1016/S1322 $-7696(08) 60491-6$

[48] Johnson B. The intentional mentor: Strategies and guidelines for the practice of mentoring. Professional Psychology - Research \& Practice. 2002; 33(1): 88-96. https://doi.org/10.1037/0735 $-7028.33 .1 .88$

[49] Gibson SK. Being mentored: The experience of women faculty. Journal of Career Development. 2004; 30(3): 173-188. https: //doi.org/10.1023/B: JOCD. 0000015538.41144 .2

[50] Sambunjak D, Straus S, Marušić A. Mentoring in academic medicine: a systematic review. JAMA. 2006; 296(9): 1103-1115. http://dx .doi.org/10.1001/jama.296.9.1103 